\title{
ARTICLE Effects of MDMA on attention to positive social cues and pleasantness of affective touch
}

\author{
Anya K. Bershad ${ }^{1,2}$, Leah M. Mayo $\mathbb{D}^{3}$, Kathryne Van Hedger ${ }^{4}$, Francis McGlone ${ }^{5,6}$, Susannah C. Walker ${ }^{5}$ and Harriet de Wit ${ }^{1}$
}

The psychostimulant drug \pm 3 ,4-methylenedioxymethamphetamine (MDMA) reportedly produces distinctive feelings of empathy and closeness with others. MDMA increases social behavior in animal models and has shown promise in psychiatric disorders, such as autism spectrum disorder (ASD) and post-traumatic stress disorder (PTSD). How it produces these prosocial effects is not known. This behavioral and psychophysiological study examined the effects of MDMA, compared with the prototypical stimulant methamphetamine (MA), on two measures of social behavior in healthy young adults: (i) responses to socially relevant, "affective" touch, and (ii) visual attention to emotional faces. Men and women $(N=36)$ attended four sessions in which they received MDMA $(0.75$ or $1.5 \mathrm{mg} / \mathrm{kg}), \mathrm{MA}(20 \mathrm{mg})$, or a placebo in randomized order under double-blind conditions. Responses to experienced and observed affective touch (i.e., being touched or watching others being touched) were assessed using facial electromyography (EMG), a proxy of affective state. Responses to emotional faces were assessed using electrooculography (EOG) in a measure of attentional bias. Subjective ratings were also included. We hypothesized that MDMA, but not MA, would enhance the ratings of pleasantness and psychophysiological responses to affective touch and increase attentional bias toward positive facial expressions. Consistent with this, we found that MDMA, but not MA, selectively enhanced ratings of pleasantness of experienced affective touch. Neither drug altered the ratings of pleasantness of observed touch. On the EOG measure of attentional bias, MDMA, but not MA, increased attention toward happy faces. These results provide new evidence that MDMA can enhance the experience of positive social interactions; in this case, pleasantness of physical touch and attentional bias toward positive facial expressions. The findings are consistent with evidence that the prosocial effects are unique to MDMA relative to another stimulant. Understanding the behavioral and neurobiological processes underlying the distinctive social effects of MDMA is a key step to developing the drug for psychiatric disorders.

Neuropsychopharmacology (2019) 44:1698-1705; https://doi.org/10.1038/s41386-019-0402-z

\section{INTRODUCTION}

The psychostimulant drug $\pm 3,4$-methylenedioxymethamphetamine (MDMA) has been labeled an "empathogen-entactogen," and reportedly increases feelings of empathy and interpersonal closeness [1, 2]. Laboratory studies in both rodents and healthy human volunteers support these reports. In rats, MDMA produces a distinctive tactile behavior of "adjacent lying" between pairs of animals, and it increases affiliative behaviors in non-human primates and cephalopods [3-6]. In humans, the drug increases self-report ratings of sociability and friendliness [7-9], as well as feelings of "trust," "openness," and "closeness with others" [10]. Notably, the effects of MDMA are distinct from the effects of other pharmacologically related psychostimulant drugs [11]. To understand the psychological processes, and ultimately the neurobiological processes, that produce these distinctive social effects of MDMA, we and others have investigated the influence of the drug on standardized behavioral tasks believed to model aspects of social behavior (for reviews see refs. [11, 12]). In this placebo-controlled study, we investigated the effects of MDMA and the prototypical stimulant methamphetamine (MA) on two dimensions of responses to social stimuli: visual attention to faces expressing emotion and on a novel measure of affective responses to social touch.

The sense of touch is central to social communication [13]. However, the sense of touch has received little attention in human psychopharmacology. Of particular interest to social communication is "affective" touch, a socially relevant form of touch that is experienced as hedonically pleasant. Affective touch is believed to be mediated by activation of C-tactile (CT) afferents, located on non-glabrous (hairy) skin, and it is distinct from the ability to detect touch, which is processed via $A \beta$ fibers [14, 15]. The CT afferents show an inverted U-shaped firing frequency, with the touch velocities between 1 and $10 \mathrm{~cm} / \mathrm{s}$ ("CT-optimal"), eliciting greater firing frequency than either faster or slower velocities. Corresponding with this, self-reported ratings of touch pleasantness follow this same inverted U-shape, with CT-optimal velocities rated as more pleasant than either faster or slower velocities [16], and pleasantness ratings are positively correlated with CT-fiber firing frequency. Psychophysiological responses to touch, such as facial electromyography (EMG), follow a similar trend, where

${ }^{1}$ Department of Psychiatry and Behavioral Neuroscience, University of Chicago, Chicago, IL, USA; ${ }^{2}$ University of Chicago Interdisciplinary Scientist Training Program, University of Chicago, Chicago, IL, USA; ${ }^{3}$ Center for Social and Affective Neuroscience, Department of Clinical and Experimental Medicine, Linköping University, Linköping, Sweden; ${ }^{4}$ Department of Clinical Neurological Sciences, University of Western Ontario, London, ON, UK; ${ }^{5}$ School of Natural Sciences and Psychology, Liverpool John Moores University, Liverpool, UK and ${ }^{6}$ Institute of Psychology, Health \& Society, University of Liverpool, Liverpool, UK

Correspondence: Harriet de Wit (hdew@uchicago.edu)

Received: 30 November 2018 Revised: 17 April 2019 Accepted: 22 April 2019

Published online: 30 April 2019 
non-optimal velocities elicit an increase in a negative effect that is mitigated by putative CT-afferent activation during CT-optimal touch $[17,18]$, and CT-optimal touch may enhance positive facial muscle responses [19]. Thus, this CT-firing mediated affective touch influences both the self-reported hedonic experience as well as the implicit facial muscle activity indicative of a positive affective response.

The positive hedonic qualities of affective touch may be due, in part, to unique neural and molecular underpinnings. CT-preferred stimulation activates the dorsal posterior insula and orbitofrontal cortex and is related to the pleasurableness of the experience $[15,20-24]$. The pleasantness of CT-optimal touch has been proposed to be related to increased levels of serotonin and oxytocin [25-28], both of which are influenced by MDMA. Interestingly, recreational MDMA users report that MDMA increases the pleasantness of social touch [29].

In the tasks used in this study, we included both CT-optimal ("slow"; $3 \mathrm{~cm} / \mathrm{s}$ ) and non-optimal ("fast"; $30 \mathrm{~cm} / \mathrm{s}$ ) stroking. In one task, we measured how people responded to the experience of receiving CT-optimal and non-optimal touch. In a second task, we assessed how participants responded to the mere observation of others being touched, both at CT-optimal $(3 \mathrm{~cm} / \mathrm{s})$ and nonoptimal (fast, $30 \mathrm{~cm} / \mathrm{s}$; static, $0 \mathrm{~cm} / \mathrm{s}$ ) velocities. Several previous studies have reported that vicarious ratings of moving touch show the same relationship to stroking velocity as directly felt touch [30-32]. In both tasks, we hypothesized that MDMA, but not MA, would increase perceived pleasantness of touch at the CT-optimal velocity.

One index of potential drug-induced changes in social function is the ability of the drug to alter attention to positive versus negative social cues. To examine this idea, we assessed the effects of the drug on participants' visual attention to images of faces with varying emotional expressions, from positive (happy) to negative (fearful, sad, and angry). We measured attention by recording eye movements in a dot-probe task while subjects were shown two faces, either neutral or emotional, on two sides of the screen. This task has been shown to be sensitive to drug effects [33], and to psychiatric symptoms related to social function: individuals with social anxiety exhibit increased vigilance toward threatening faces [34]. Conversely, some pharmacological manipulations, such as the administration of intranasal oxytocin, have been reported to enhance attention bias toward happy faces [35]. The findings regarding the effects of intranasal oxytocin on attention bias have been mixed, however. Domes et al. reported increased attention bias to positive stimuli but no effects on negative stimuli, whereas Kim et al. [36] reported reduced attention toward negative expressions with no effect on positive expressions. It has been been suggested that MDMA produces prosocial effects by altering oxytocinergic signaling [37], and in line with the effects of oxytocin on attention bias, we hypothesized that if MDMA is modifying attention through an oxytocinergic mechanism, then MDMA, but not MA [11], would positively bias visual attention.

Thus, this study was designed to extend our understanding of how MDMA produces prosocial effects, and how these effects differ from those of a prototypic psychostimulant. The study was conducted in a laboratory setting under placebo-controlled double-blind conditions with healthy adult volunteers.

\section{METHODS}

Study design

This study used a four-session, within-subjects, double-blind design in which young adults received placebo, $0.75 \mathrm{mg} / \mathrm{kg}$ MDMA, $1.5 \mathrm{mg} / \mathrm{kg}$ MDMA, or $20 \mathrm{mg}$ of methamphetamine (MA) in randomized order during four 4-h laboratory sessions. Outcome measures included subjective ratings of mood states and touch stimuli, psychophysiological responses, and visual attention to emotional faces.

\section{Participants}

Healthy volunteers $(N=36)$ aged $18-40$ years were recruited from the University of Chicago and the surrounding area. We recruited participants who reported between 4 and 40 uses of MDMA in their lifetime. At a screening session, participants underwent a physical and psychiatric screening, which included an in-person psychiatric interview and a detailed drug use history questionnaire, and electrocardiogram. Exclusion criteria were current diagnoses of psychiatric disorders, including major depression (DSM 2015 [38]), serious medical condition, history of cardiac or liver disease, current or past substance abuse, individuals regularly using any contraindicated medications, and individuals with a previous negative reaction to MDMA. Women who were pregnant, planning to become pregnant, or lactating were excluded. Inclusion criteria were fluency in English, a minimum of a high school education, and BMl between 19 and $30 \mathrm{~kg} / \mathrm{m}^{2}$.

\section{Study drugs}

MDMA in powdered form $(0.75$ or $1.5 \mathrm{mg} / \mathrm{kg}$ ) or MA $(20 \mathrm{mg})$ was placed in opaque-size 00 capsules with lactose (USP) filler. Placebo capsules contained only lactose. These doses have previously been safely administered to healthy human participants [39-41].

\section{Session procedures}

Orientation. During a 1-h orientation session, participants were provided with information about the study and gave informed consent. The study was approved by the University of Chicago Institutional Review Board. Participants were told that the goal of the study was to examine the effects of drugs on mood, perceptions, and behavior. To minimize expectancy effects, they were informed that they could receive a stimulant drug such as MDMA or methamphetamine, a sedative drug such as valium, a cannabinoid such as marijuana, or placebo.

Study sessions. Participants completed four 4.5-h sessions from 9:00 am to $1: 30 \mathrm{pm}$. The sessions were separated by at least 2 days and were conducted in a comfortably furnished room in a research laboratory containing chairs, a desk, computer, a television, video player, and reading materials. During the sessions, the participants were allowed to watch movies, read, or relax when not completing study questionnaires or tasks. Subjects were asked to abstain from drugs and alcohol for $48 \mathrm{~h}$ before each session, and compliance was verified at the start of each session with a urine drug (ToxCup, Branan Medical Corporation) and breathalyzer tests (Alcosensorlll, Intoximeters). Women were also tested for pregnancy before each session (AimStickPBD, hCG professional, Craig Medical Distribution). After these tests, participants completed baseline measures for their mood and cardiovascular state (see below for mood and cardiovascular measures). At 9:30 am, participants took a capsule containing MA $(20 \mathrm{mg})$, MDMA ( 0.75 or $1.5 \mathrm{mg} / \mathrm{kg})$, or placebo. Participants then relaxed for $1 \mathrm{~h}$, and subjective and cardiovascular measures were collected again at 10:00 and 10:30 am. At 10:45 am, psychophysiological sensors were placed to collect facial EMG and EOG data, and participants completed the attention bias task, the observed touch task, and experienced touch task in randomized order. At 11:45 am, the psychophysiological sensors were removed and subjective and cardiovascular measures were re-assessed at 12:30 and 1:00 pm. Participants then completed an end-of-session questionnaire and left the laboratory. After completing all sessions, participants were provided with information on which drug or placebo they ingested at each of their study sessions, and were permitted to ask any questions they had about the study during a conversation with the researchers. Participants also received monetary compensation for their time. 


\section{Subjective questionnaires}

Drug effect questionnaire (DEQ). The DEQ consists of five questions on a visual analog scale assessing subjective drug effects. Subjects were asked to rate the extent they felt about a drug effect, whether they liked or disliked the drug effect, if they felt high, and if given a choice, whether they would want more of the drug.

Visual analog scales (VAS). VAS consisted of adjective-tapping constructs that have previously been validated as sensitive to MDMA effects [40, 42], including words such as "Sociable," "Confident," "Lonely," "Playful," "Dizzy," "Loving," "Friendly," and "Restless" (Supplementary Table 1).

End-of-session questionnaire (ESQ). This is a short questionnaire addressing which drug the participants believed they received and how much they would like to take the drug again.

\section{Behavioral tasks}

Experienced touch task. In this task, touch was administered using a 5-cm-wide goat hair brush applied to a $9-\mathrm{cm}$ section of the forearm. We used a brush rather than hand touching (i.e., skin-toskin contact) to control for variation in skin temperature [43]. We used a handheld brush, as previous reports indicate no difference in ratings of tactile stimulation delivered manually versus a forcecontrolled robot [44]. Participants were instructed to look straight ahead at a fixation cross presented on a computer screen throughout the brushing trials to avoid providing any visual cues regarding touch administration. The experimenter wore headphones to receive metronomic audio cues for consistency in brushing velocity. The task consisted of four blocks of eight trials each (32 total trials). Each trial consisted of $6 \mathrm{~s}$ of touch stimulation followed by ratings. Each block contained eight trials, four of each velocity: $3 \mathrm{~cm} / \mathrm{s}$ ("slow") and $30 \mathrm{~cm} / \mathrm{s}$ ("fast"). Velocity order within each block pseudorandomized but was not repeated more than three times. Affective responses to the brushing were measured using facial EMG (described below) and self-report ratings of pleasantness. Ratings were conducted during inter-trial intervals using a 7-point Likert scale ranging from 1 (extremely unpleasant) to 7 (extremely pleasant). A 1-2 -s variable inter-trial interval (ITI) separated each rating, with the final rating followed by a 7-9-s variable ITI before the next trial began.

Observed touch task. In the observed touch task, participants viewed 6-s videos depicting a left arm (dorsal and ventral forearm and upper arm), back, or palm resting on a white background, being stroked by a hand at a rate of 0 (static), 3 or $30 \mathrm{~cm} / \mathrm{s}$. The task consisted of two repetitions of blocks of 60 trials. A trial consisted of viewing touch being delivered to a given location [back, medial forearm, lateral forearm, upper arm, or palm] at one of the three velocities [0 cm/s ("static"), $3 \mathrm{~cm} / \mathrm{s}$ ("slow"), or $30 \mathrm{~cm} / \mathrm{s}$ ("fast")]. Within a block, each location $\times$ velocity combination was presented four times in a pseudorandomized order (no more than three consecutive identical locations or velocities). Facial muscle activity during video presentation was assessed via facial EMG, as described below. A 7.5-8.5-s ITI followed each video, during which time participants rated the pleasantness of the video, similar to the experienced touch task. For the analysis presented here, we used only ratings of touch to the three surfaces of the arm to be consistent with the experienced touch task. Data for all locations are presented in Supplementary Table 2.

Facial electromyography (EMG). Emotional reactivity to touch tasks was determined through EMG of corrugator and zygomatic musculature. EMG measures of these facial muscles' activity are indicative of affective responses to emotional stimuli and can provide another measure of emotional reactivity beyond subjective reports alone $[45,46]$. Highly positive stimuli increase activity in the zygomatic, or "smile" muscle, and relax the corrugator ("frown") muscle. Negative stimuli activate the corrugator muscle. EMG was recorded from 4- $\mathrm{mm} \mathrm{Ag/AgCl}$ electrodes precisely placed on the cheek and left brow. An 8-mm Ag/AgCl ground sensor was placed on the subject's forehead. Signals were amplified, filtered through a $10-$ to $500-\mathrm{Hz}$ band pass, digitized, re-filtered, rectified, and then integrated over $20 \mathrm{~ms}$ using EMG100C amplifiers, an MP150 Data Acquisition System, and Acqknowledge software from Biopac Systems (Goleta).

Attention bias task (ABT). The ABT was adapted from Garner et al. [47] and presented using E-Prime 2.0 software. Participants were shown pairs of faces, one on each side of the screen. Each pair contained one neutral face and one emotional expression face using the same actor. The emotional expressions were collected from the standardized Karolinska set [48]. Each face pair presentation consisted of a 1-s pre-picture fixation, then a 2-s picture presentation. To distract the participants from the purpose of the task, a probe (either a square or a circle) was presented following the 2-s display of the face pair, and the participants were asked to indicate whether the shape presented was a square by pressing one keyboard key or if it was a circle by pressing a different key. After a response, or $10 \mathrm{~s}$ elapsed, an inter-trial interval of 750-1250 ms began, followed by another trial. EOG was used to quantify which face was initially fixated on in each trial and was collected with $4-\mathrm{mm} \mathrm{Ag} / \mathrm{AgCl}$ electrodes attached $1.5 \mathrm{~cm}$ from the outer canthus of each eye. The application and data collection process was the same as the EMG application and collection detailed above. Trained, blinded scorers discarded trials in which the gaze was not centrally fixed prior to the trial, the initial fixation was $<100 \mathrm{~ms}$ after picture onset, or noise obscured eye movements. Data were excluded for sessions prior to which subjects had $4 \mathrm{~h}$ or less sleep, given the effects of sleep deprivation on attention [49].

Cardiovascular measures

Heart rate and blood pressure were monitored with portable monitors (Omron 10 Plus, Omron Healthcare) five times during the session $(-15,30,60,180$, and 240 min post drug administration). Mean arterial pressure was calculated using the formula: MAP = (systolic BP $+2 \times$ diastolic BP)/3.

Statistical analysis

Analyses were conducted using SPSS. Missing cases (due to equipment malfunction or other data collection problems) were deleted list wise, which led to smaller sample sizes for some analyses. For subjective measures collected at multiple time points, peak or peak change from baseline was calculated for the purpose of analysis and concise representation in data tables. Subjective effects of the drug were assessed using repeated measures analysis of variance (ANOVA), with dose as a within-subject factor. MDMA vs. placebo and MA vs. placebo were analyzed separately, with linear contrasts used for the MDMA analysis, given the two doses. Subjective and psychophysiological data from the touch tasks were analyzed with repeated measure ANOVAs, with dose and touch velocity as within-subject factors. The ABT was also analyzed with repeated measure ANOVAs, with dose as a within-subject factor. The significant main effects and interactions were followed with post hoc $t$ tests corrected for multiple comparisons with the Benjamini-Hochberg procedure with a false discovery rate of 0.05 .

\section{RESULTS}

\section{Demographics}

The subjects were men and women with a mean age of 24.8 $( \pm 4.2)$, most of whom were Caucasian $(61 \%)$ with some postgraduate education. They reported using MDMA an average 
of $11.1 \pm 9.8$ times in their lifetime. Demographic characteristics are summarized in Table 1.

Subjective drug effects

Both doses of MDMA and the single dose of MA increased participants' reports of "feel drug" [MDMA $F(1,35)=144.53$, $p<0.001$; both doses vs. placebo $p<0.001$; $M A F(1,35)=18.2$, $p<0.001$; Supplementary Table 1], "like drug" [MDMA $\mathrm{F}(1,35)=$ $68.55, p<0.001$; both doses vs. placebo $p<0.001 ; \operatorname{MA~F}(1,35)=25.0$, $p<0.001$ ], and "feel high" [MDMA $\mathrm{F}(1,35)=109.22, p<0.001$; $0.75 \mathrm{mg} / \mathrm{kg}$ vs. placebo $p<0.01,1.5 \mathrm{mg} / \mathrm{kg}$ vs. placebo $p<0.001$; MA $F(1,35)=17.5, p<0.001]$. The higher dose of MDMA increased ratings of "dislike drug" [MDMA $\mathrm{F}(1,35)=13.4, p<0.01 ; 1.5 \mathrm{mg}$ vs. placebo $p<0.01]$, and all doses increased ratings of "want more"

\begin{tabular}{|lr|}
\hline Table 1. Participant demographics & \\
\hline Sex & \\
$\quad$ Male & $18(50)$ \\
$\quad$ Female & $18(50)$ \\
Race & \\
$\quad$ Caucasian & $22(61)$ \\
$\quad$ African American & $5(14)$ \\
$\quad$ Asian & $5(14)$ \\
$\quad$ Other & $4(11)$ \\
Age & $24.8(4.2)$ \\
BMI & $23.3(1.1)$ \\
Education in years & $15.2(1.5)$ \\
Substance use & \\
$\quad$ Alcoholic drinks/week & $3.7(2.3)$ \\
$\quad$ MDMA (number of times used) & $11.1(9.8)$ \\
\hline Demographic and drug use characteristics reported as N (\%) or M (SD) \\
\hline
\end{tabular}

\section{a Ratings of experienced touch}

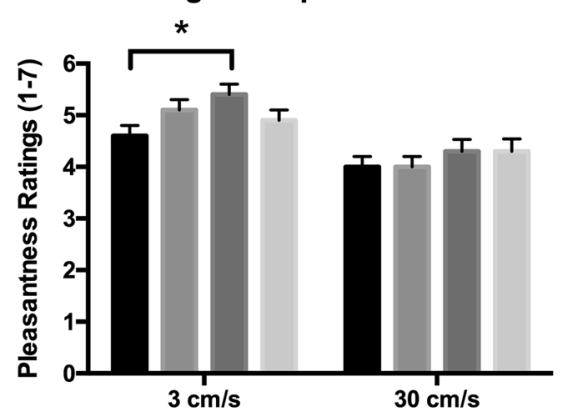

Placebo $0.75 \mathrm{mg} / \mathrm{kg}$ MDMA $1.5 \mathrm{mg} / \mathrm{kg}$ MDMA $20 \mathrm{mg} \mathrm{MA}$
[MDMA $\mathrm{F}(1,35)=13.4, p<0.001 ; 0.75 \mathrm{mg} / \mathrm{kg}$ vs. placebo $p<0.01$ and $1.5 \mathrm{mg}$ vs. placebo $p<0.001 ; \mathrm{MA} \mathrm{F}(1,35)=27.5, p<0.001]$.

The higher dose of MDMA increased ratings of "anxious" [MDMA $\mathrm{F}(1,35)=6.78, p<0.05 ; 1.5 \mathrm{mg} / \mathrm{kg}$ vs. placebo $p<0.05$ ]. Both the higher dose of MDMA and MA increased ratings of "insightful" [MDMA $\mathrm{F}(1,35)=6.78, p<0.05 ; 1.5 \mathrm{mg} / \mathrm{kg}$ vs. placebo $p<0.05 ; \mathrm{MA}$ $\mathrm{F}(1,35)=11.86, p<0.01]$ and "stimulated" [MDMA $\mathrm{F}(1,35)=10.25$, $p<0.01 ; 1.5 \mathrm{mg} / \mathrm{kg}$ vs. placebo $p<0.01 ; \mathrm{MA} \mathrm{F}(1,35)=5.22, p<0.05$ ]. MA significantly increased ratings of "sociable" $[M A F(1,35)=6.40$, $p<0.05]$ and "lonely" [MA $\mathrm{F}(1,35)=8.42, p<0.01]$. MA increased ratings of "playful," and $1.5 \mathrm{mg} / \mathrm{kg}$ MDMA tended to do the same [MDMA $\mathrm{F}(1,35)=5.8, p<0.05 ; 1.5 \mathrm{mg} / \mathrm{kg}$ vs. placebo $p=0.06 ; \mathrm{MA}$ $\mathrm{F}(1,35)=4.6, p<0.05]$. MA increased ratings of "loving" [MA $\mathrm{F}(1,35)=5.58, p<0.05]$. All doses increased ratings of "restless" [MDMA $\mathrm{F}(1,35)=15.01, p<0.01 ; 0.75 \mathrm{mg} / \mathrm{kg}$ vs. placebo $p<0.01$ and $1.5 \mathrm{mg} / \mathrm{kg}$ vs. placebo $p<0.01 ; \mathrm{MA} \mathrm{F}(1,35)=1.44, p<0.01$ ]. None of the doses significantly affected ratings of confident, friendly, or dizzy (see Supplemental Table 1).

Cardiovascular drug effects

MA and both doses of MDMA significantly increased MAP and heart rate [MAP: MDMA $F(2,70)=24.6, p<0.001$; placebo vs. $0.75 \mathrm{mg} / \mathrm{kg}$ MDMA, $p<0.01$; placebo vs. $1.5 \mathrm{mg} / \mathrm{kg}$ MDMA, $p<0.001$; MA $\mathrm{F}(1,35)=30.9, p<0.001$; heart rate: $\operatorname{MDMA} \mathrm{F}(2,70)=27.6, p<0.001$; placebo vs. $0.75 \mathrm{mg} / \mathrm{kg}$ MDMA, $p<0.01$; placebo vs. $1.5 \mathrm{mg} / \mathrm{kg}$ MDMA, $p<0.01$; MA F $(1,35)=25.7, p<0.001]$.

Experienced touch task

Self-report: Ratings of pleasantness differed across the two velocities [Fig. 1a; $F(1,35)=38.0, p<0.001$ ], such that CT-optimal (slow) touch was always rated as more pleasant than non-optimal touch. Further, both doses of MDMA increased self-reported ratings of pleasantness $[F(2,70)=7.27, p<0.05]$ for slow touch. Follow-up tests revealed that ratings of slow touch were marginally enhanced following the low dose of MDMA $(p=0.051)$ and significantly enhanced following the high dose of MDMA ( $p=$ 0.012 ). Analysis of the MA sessions revealed no significant main

\section{b}

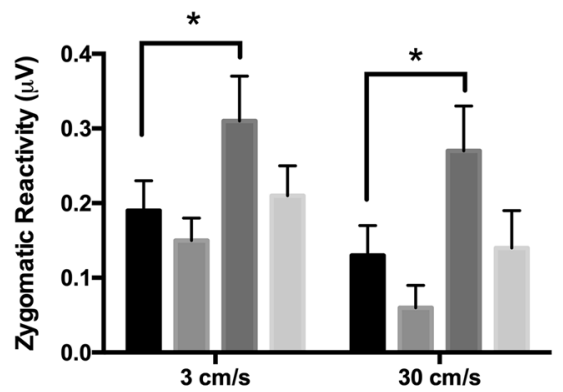

Placebo $0.75 \mathrm{mg} / \mathrm{kg}$ MDMA $1.5 \mathrm{mg} / \mathrm{kg}$ MDMA 20mg MA

C EMG reponses to experienced touch

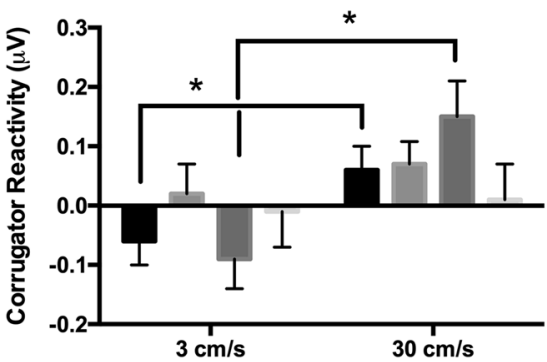

Placebo

$0.75 \mathrm{mg} / \mathrm{kg}$ MDMA

$1.5 \mathrm{mg} / \mathrm{kg}$ MDMA 20mg MA

Fig. 1 Effects of MDMA, MA, and placebo on a pleasantness ratings, $\mathbf{b}$ zygomatic reactivity, and c corrugator reactivity to CT-optimal and nonoptimal experienced touch. ${ }^{*}$ indicates a significant difference between drug and placebo, $p<0.05$. Statistical analysis was conducted on MDMA vs. placebo and MA vs. placebo separately, but doses are depicted together for the sake of clarity 


\section{2}

effect of the drug (Supplementary Table 1). There was no significant effect of drug on ratings of non-optimal (fast) touch.

Zygomatic. There was a linear dose effect of MDMA on zygomatic reactivity at both velocities [Fig. $1 \mathrm{~b} ; \mathrm{F}(1,33)=8.04$, $p<0.01]$. There was a trend toward the main effect of velocity $[F(1,33)=3.07, p=0.09]$, but this did not reach significance. There was no significant effect of MA on zygomatic responses, or significant drug $\times$ velocity interaction (Supplementary Table 1 ).

Corrugator. Consistent with prior findings, fast touch produced greater muscle activity than slow touch [Fig. 1c; main effect of velocity; $F(1,31)=13.9, p<0.01$, but the drug/dose had no significant effect on this measure $(F(1,32)=0.25, p=0.66)$. Post hoc tests revealed that fast touch $(30 \mathrm{~cm} / \mathrm{s})$ elicited greater corrugator activity than slow touch (CT-optimal; $3 \mathrm{~cm} / \mathrm{s}$ ) during the placebo session $(p=0.013)$ and high-dose MDMA session $(p=$ $0.007)$, but elicited no significant difference during the low-dose MDMA or MA sessions. There was no significant effect of MA on corrugator reactivity (Supplementary Table 1).

\section{Observed touch task}

Self-report: Videos of slow touch were rated as more pleasant than fast and static touch, but there was no significant difference in ratings of static and fast touch [Supplementary Fig. $1 A ; F(2,70)=$ $21.4, p<0.001 ; 3 \mathrm{~cm} / \mathrm{s}$ vs. $0 \mathrm{~cm} / \mathrm{s}, p<0.001 ; 3 \mathrm{~cm} / \mathrm{s}$ vs. $30 \mathrm{~cm} / \mathrm{s}, p<$ $0.001]$. There was no significant main effect of drug/dose or drug/ dose $\times$ velocity interaction (Supplementary Tables 1 and 2).

Zygomatic. There were no significant effects of velocity or drug on zygomatic reactivity (Supplementary Fig. 1B and Supplementary Table 1), though there was a trend toward a linear effect of MDMA, as seen with the experienced touch task, such that MDMA increased zygomatic activity $[\mathrm{F}(1,30)=3.83, p=0.06]$.

Corrugator. There were no significant effects of velocity or drug on corrugator reactivity (Supplementary Fig. 1C and Supplementary Table 1)

\section{Attention bias task}

At $1.5 \mathrm{mg} / \mathrm{kg}$, MDMA increased the total number of times participants looked toward happy faces before neutral faces (i.e., orienting attention), but did not affect gazes toward other emotion types [Fig. 2; $F(1,29)=4.45, p<0.05$; placebo vs. $1.5 \mathrm{mg} / \mathrm{kg}$ MDMA, $p<0.05$; MA $F(1,29)=0.32, p=0.58]$. MA significantly increased the first gaze toward angry faces $[F(1,29)=5.1, p<0.05]$, but MDMA did not $[\mathrm{F}(1,29)=1.50, p=0.23]$. Otherwise, neither MDMA nor MA significantly affected initial gazes toward fear and sadness [MDMA $\mathrm{F}(1,29)=0.38, p=0.54 ; \operatorname{MA~} \mathrm{F}(1,29)=0.15, p=0.7 ; \operatorname{MDMA} \mathrm{F}(1,29)=$ $0.25, p=0.62 ; \mathrm{MA} \mathrm{F}(1,29)=0.09, p=0.77]$.

\section{Visual attention to facial expressions}

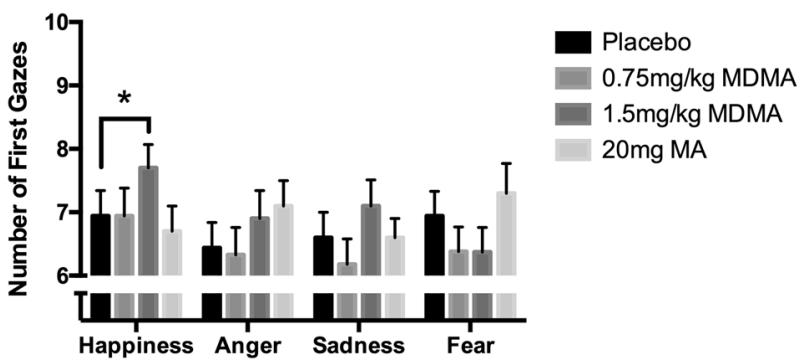

Fig. 2 Mean ( \pm SEM) number of initial gazes toward emotional facial expressions after MDMA, MA, or placebo, shown for each emotion depicted. Asterisk indicates a significant difference between drug and placebo, $p<0.05$
Drug identifications

During the placebo session, 23 participants (63.9\%) correctly guessed what they had received. The others guessed that they had taken valium (22.2\%), MDMA (5.6\%), MA (5.6\%), or marijuana (2.8\%). During the MA session, nine participants (25\%) correctly guessed what they had received. The rest guessed that they had taken valium (5.6\%), MDMA (38.9\%), marijuana (11.1\%), or placebo (19.4\%). During the $0.75 \mathrm{mg} / \mathrm{kg}$ MDMA session, 17 participants (47.2\%) correctly guessed what they had received. The rest guessed that they had taken valium (25\%), MA (5.6\%), marijuana $(8.3 \%)$, or placebo (13.9\%). During the $1.5 \mathrm{mg} / \mathrm{kg}$ MDMA session, 25 participants $(69.4 \%)$ correctly guessed what they had received. The rest guessed that they had taken valium (11.1\%), MA (16.7\%), or placebo $(2.8 \%)$. To assess the effects of expectancy on responses to affective touch, we compared pleasantness ratings for subjects who believed that they had received MDMA after ingesting MA to those who believed that they had received anything else, and ratings were not significantly different $[\mathrm{t}(34)=$ $1.41, p=0.17]$

\section{DISCUSSION}

In this study, we investigated the effects of MDMA and the prototypic stimulant MA, compared with placebo, on responses to two psychophysiological processes that may mediate the unique prosocial effects of MDMA: a novel measure of affective touch and visual attention to emotional faces. In accordance with our hypothesis, MDMA dose-dependently increased pleasantness ratings of affective touch at the CT-optimal frequency, without significantly affecting pleasantness of non-optimal touch. MA did not have this effect. Further and in line with these results, MDMA tended to increase pleasantness ratings for observed touch, although these effects were not significant. MDMA tended to increase zygomatic reactivity to touch at both frequencies, but did not significantly affect corrugator activity. MDMA also enhanced attention bias toward happy faces, but not significantly to other emotions, whereas MA did not have this effect. Taken together, these findings advance our understanding of how MDMA influences social interaction.

We examined affective responses to soft touch, using both subjective ratings and psychophysical indices (zygomatic and corrugator muscle activity). Touch is a relatively recent and promising measure of social affective response. CT-optimal touch refers to physical touch that is between 1 and $10 \mathrm{~cm} / \mathrm{s}$ applied to hairy skin, and can be contrasted to non-optimal touch, which occurs outside this narrow frequency range, is rated as less pleasant than CT-optimal touch [16], and induces corrugator activation [17]. Further, in another study, participants were asked to stroke other individuals with whom they have intimate relationships (partners and babies) at any velocity, and reliably chose CT-optimal velocities, which they do not do when asked to stroke a wooden arm [50]. CT-optimal touch preferentially activates the dorsal posterior insula (as compared with nonoptimal touch), a region involved in affective tactile processing [51]. Interestingly, this region is also implicated in increased resting state connectivity induced by MDMA (Bjornsdotter et al. 2017 [52]). This suggests that MDMA may act directly upon the neural circuits involved in responding to affective touch, which may explain subjective reports of increased touch pleasantness. Our results that MDMA enhances pleasantness ratings of affective touch are consistent with this evidence.

In our study, MDMA increased zygomatic reactivity to both velocities of touch. This finding was unexpected, and may have been related to bruxism, a common side effect of MDMA [53]. Although the facial EMG electrodes were placed to target the zygomatic muscle, there may have been some contamination from the masseter muscle, which could explain the relatively nonspecific increase in zygomatic activation to both velocities of 
touch at the higher dose of MDMA. In our study, for the high-dose MDMA session, zygomatic responses during slow touch were correlated with pleasantness ratings $(r=0.54, p<0.01)$, suggesting an alternative explanation of our results (beyond bruxism), that MDMA enhances positive psychophysiological responses to both CT-optimal and non-optimal touch.

The mechanisms by which MDMA alters responses to affective touch are unknown. It has been suggested that MDMA produces prosocial effects by altering serotonergic $[54,55]$ and oxytocinergic signaling [37]. Changes in serotonergic signaling have been linked to changes in social behavior, and MDMA may lead to such changes $[32,56]$. Several studies have reported that MDMA increases plasma oxytocin [57-60], even to levels that exceed those attained by the administration of intranasal oxytocin itself $[42,59]$. Some studies [57], but not others [61] find that plasma oxytocin levels are correlated with the prosocial effects of MDMA. A handful of studies have investigated the effects of intranasal oxytocin on the pleasantness of affective touch, but the findings have been inconclusive $[62,63]$, and there is controversy about the amount of oxytocin that reaches the brain after intranasal administration [64]. MDMA has been shown to increase plasma oxytocin significantly more than the administration of intranasal oxytocin itself [59]. Notably, MA, which did not increase ratings of pleasantness in this study, also does not increase plasma concentrations of oxytocin [65].

The increase in oxytocin levels observed after the administration of MDMA may be secondary to the drug's effects on the serotonergic system. Serotonin activates $5 \mathrm{H}-\mathrm{T}_{1 \mathrm{~A}}$ receptors in the hypothalamus, which then leads to the release of oxytocin into the blood [37]. Pharmacologic investigations into the role of the serotonergic system in producing the "empathogenic" effects of MDMA have provided some new evidence. While pretreatment with a selective serotonin reuptake inhibitor (SSRI) attenuates some subjective effects of MDMA, it is not clear if SSRIs specifically attenuate the social effects of MDMA [66]. Liechti et al. [67] reported that the SSRI citalopram reduced the effects of MDMA on ratings of self-confidence and extraversion but it did not reduce ratings of emotional sensitivity and excitability. Another study reported that the SSRI paroxetine dampened the effects of MDMA on both social and nonspecific measures of euphoria (i.e., "very happy," and "more positive view about things" [68]).

Beyond the serotonergic system, noradrenergic and dopaminergic mechanisms may also contribute to some of the subjective effects of MDMA. Duloxetine, a serotonin-norepinephrine reuptake inhibitor, and reboxetine, a norepinephrine reuptake inhibitor, reportedly attenuate self-reported prosocial effects of the drug $[69,70]$. MDMA can increase dopamine both directly and indirectly, and some of the positive mood effects of MDMA may be blocked by the administration of haloperidol [66]. In sum, serotonin, norepinephrine, and dopamine release likely contribute to some of the self-reported social effects of MDMA in humans, and the role of each neurotransmitter system in producing the effects observed in this study is a topic for further research.

The finding that MDMA enhances the pleasantness of social touch has clinical implications for treatment of psychiatric disorders. MDMA has been used as an adjunct to psychotherapy in patients with PTSD [71], and phase III clinical trials are underway to test its efficacy. Whether touch plays a role in this context remains to be determined. MDMA is also being considered in the treatment of autism spectrum disorder (ASD). It has been shown to decrease social anxiety in autistic adults when used in combination with psychotherapy [72, 73]. Interestingly, a key symptom of ASD is altered touch processing $[63,74,75]$ : those with autistic traits find that affective touch is less pleasant than healthy controls, and they exhibit reduced activity in brain regions sensitive to CT-optimal touch [75, 76]. Thus, MDMA may act to boost responsiveness to CT stimulation in these populations, and help to normalize affective responses to touch.

The other finding in this study was that MDMA enhanced attention bias toward happy faces, as measured by eye movements. Previously, we showed that MDMA increased positive ratings of images with social content [41], and others showed that it increases some measures of emotional empathy [77-79]. Other reports suggest that MDMA positively biases interpretation of emotional facial expressions, by enhancing identification of positive expressions, interfering with identification of negative expressions, or both $[9,42,58,80]$. Negative processing bias has been observed in multiple psychiatric disorders [81, 82], including PTSD [83], and the positive-biasing effects of MDMA we report here may underlie its effectiveness in a therapeutic context [71, 84-86].

Our study had a number of strengths. We studied two doses of MDMA, as well as placebo and the comparison drug MA, under double-blind conditions. The participants were carefully screened to limit variability in body weight, past and recent drug use history, psychiatric symptoms, and in women, menstrual cycle phase. By administering the drug under double-blind conditions, where subjects could expect to receive a stimulant (including MDMA), a tranquilizer, or a placebo, we minimized expectancies that can contribute to responses to drugs under non-laboratory conditions $[87,88]$. The use of two doses of MDMA helps to demonstrate the sensitivity of the tasks and the pharmacological profile of the drug. The use of a comparison drug, MA, helps to distinguish the effect of MDMA from that of a prototypic psychostimulant. Finally, some of the measures used, including both subjective reports and psychophysiology, are standardized and sensitive indicators of psychoactive drug effects. The touch tasks were novel and open a new window of opportunities for psychopharmacology studies.

A few limitations of this study warrant consideration. First, the participants were healthy adult volunteers with no psychiatric pathology, and it is not known if individuals with psychiatric symptoms would respond similarly to the drug. Second, we did not obtain measures of plasma oxytocin levels, which would have enabled us to speculate about a possible mechanism for the observed effects. Further, we focused in this study on affective touch applied to non-glabrous skin areas, which are innervated with CT afferents. Follow-up studies could compare the effects of MDMA on responses to touch applied to both glabrous and non-glabrous skin. Finally, we tested the effects of MDMA in a highly controlled laboratory environment. For example, whereas the touch procedure used in this study was intended to simulate aspects of the real-world experience (e.g., social touch with a partner, family member, or other close friend), the procedure does not replace the actual experience of touch by these individuals. Both affective touch and the perception of emotional faces are likely more complex and more salient in real-world settings, and our study was unable to fully replicate these encounters.

In summary, this is the first study to examine the effects of MDMA on responses to affective touch, and to directly compare those effects with another prototypical psychostimulant, MA. We showed that MDMA selectively enhances the pleasantness of CToptimal touch, and that it biases visual attention to positive emotional faces. Both of these effects could contribute to the therapeutic efficacy of the drug. Interestingly, we compared MDMA with a psychostimulant drug without strong serotonergic effects (MA), and did not see similar effects on touch or attention. Many questions remain, including questions of the precise mechanism by which these effects occur (e.g., via serotonergic, noradrenergic, dopaminergic, and oxytocinergic signaling), and whether the effects play a role in the therapeutic benefits of the drug in clinical populations. Further research will resolve these questions. 


\section{FUNDING AND DISCLOSURE}

This work was supported by a grant from the National Institute on Drug Abuse (DA02812), and AKB was supported by a training grant from the National Institute of General Medical Sciences (2T32GM007281). The authors declare no competing interests.

\section{ACKNOWLEDGEMENTS}

We would like to thank Dr. Royce Lee for his assistance in screening subjects for this study. We would also like to thank Rick Doblin and the Multidisciplinary Association for Psychedelic Studies (MAPS) for generously supplying the MDMA for this project.

\section{ADDITIONAL INFORMATION}

Supplementary Information accompanies this paper at (https://doi.org/10.1038/ s41386-019-0402-z).

Publisher's note: Springer Nature remains neutral with regard to jurisdictional claims in published maps and institutional affiliations.

\section{REFERENCES}

1. Peroutka SJ, Newman $H$, Harris $H$. Subjective effects of 3 , 4-methylenedioxymethamphetamine in recreational users. Neuropsychopharmacology. 1988;1:273-7.

2. Siegel RK. MDMA: Nonmedical use and intoxication. J Psychoact Drugs. 1986;18:349-54.

3. Edsinger $E$, Dölen $G$. A conserved role for serotonergic neurotransmission in mediating social behavior in octopus. Curr Biol. 2018;28:3136-42.

4. Pitts EG, Minerva AR, Oliver EB, Howell L. MDMA increases affiliative behaviors and vocalizations in nonhuman primates in a 5 - $\mathrm{HT} 2 \mathrm{~A}$ receptor-dependent manner. Drug Alcohol Depend. 2017;171:e166-7.

5. Ramos L, Hicks C, Caminer A, Goodwin J, McGregor IS. Oxytocin and MDMA ('Ecstasy') enhance social reward in rats. Psychopharmacology. 2015;232:2631-41.

6. Thompson M, Hunt G, McGregor I. Neural correlates of MDMA ("Ecstasy")-induced social interaction in rats. Soc Neurosci. 2009;4:60-72.

7. Harris DS, Baggott $M$, Mendelson JH, Mendelson JE, Jones RT. Subjective and hormonal effects of 3, 4-methylenedioxymethamphetamine (MDMA) in humans. Psychopharmacology. 2002;162:396-405.

8. Bedi G, Hyman D, de Wit H. Is ecstasy an "empathogen"? Effects of \pm 3 , 4-methylenedioxymethamphetamine on prosocial feelings and identification of emotional states in others. Biol psychiatry. 2010;68:1134-40.

9. Bedi G, Phan KL, Angstadt M, de Wit H. Effects of MDMA on sociability and neural response to social threat and social reward. Psychopharmacology. 2009;207:73-83.

10. Schmid Y, Hysek CM, Simmler LD, Crockett MJ, Quednow BB, Liechti ME. Differential effects of MDMA and methylphenidate on social cognition. J Psychopharmacol. 2014;28:847-56.

11. Bershad AK, Miller MA, Baggott MJ, de Wit H. The effects of MDMA on socioemotional processing: does MDMA differ from other stimulants? J Psychopharmacol. 2016a;30:1248-58.

12. Kamilar-Britt $P$, Bedi $G$. The prosocial effects of 3, 4-methylenedioxymetham phetamine (MDMA): controlled studies in humans and laboratory animals. Neurosci Biobehav Rev. 2015;57:433-46.

13. Gallace A, Spence C. The science of interpersonal touch: an overview. Neurosci Biobehav Rev. 2010;34:246-59.

14. Abraira VE, Ginty DD. The sensory neurons of touch. Neuron. 2013;79:618-39.

15. Olausson $\mathrm{H}$, Wessberg J, McGlone F, Vallbo $\AA$. The neurophysiology of unmyelinated tactile afferents. Neurosci Biobehav Rev. 2010;34:185-91.

16. Löken LS, Wessberg J, McGlone F, Olausson H. Coding of pleasant touch by unmyelinated afferents in humans. Nat Neurosci. 2009;12:547.

17. Mayo LM, Lindé J, Olausson H, Heilig M. Putting a good face on touch: facial expression reflects the affective valence of caress-like touch across modalities. Biol Psychol. 2018;137:83-90.

18. Ree A, Mayo LM, Leknes S, Sailer U. Touch targeting C-tactile afferent fibers has a unique physiological pattern: a combined electrodermal and facial electromyography study. Biol Psychol. 2018;140:55-63.

19. Pawling R, Cannon PR, McGlone FP, Walker SC. C-tactile afferent stimulating touch carries a positive affective value. PloS ONE. 2017;12:e0173457.

20. Björnsdotter $M$, Olausson $\mathrm{H}$. Vicarious responses to social touch in posterior insular cortex are tuned to pleasant caressing speeds. J Neurosci. 2011;31:9554-62.

21. Francis S, Rolls ET, Bowtell R, McGlone F, O'Doherty J, Browning A, et al. The representation of pleasant touch in the brain and its relationship with taste and olfactory areas. Neuroreport. 1999;10:453-9.
22. McGlone F, Wessberg J, Olausson H. Discriminative and affective touch: sensing and feeling. Neuron. 2014;82:737-55.

23. Olausson H, Lamarre $Y$, Backlund H, Morin C, Wallin B, Starck G, et al. Unmyelinated tactile afferents signal touch and project to insular cortex. Nat Neurosci. 2002;5:900.

24. Perini I, Olausson H. Seeking pleasant touch: neural correlates of behavioral preferences for skin stroking. Front Behav Neurosci. 2015;9:8.

25. Trotter PD, McGlone F, McKie S, McFarquhar M, Elliott R, Walker SC, et al. Effects of acute tryptophan depletion on central processing of CT-targeted and discriminatory touch in humans. Eur J Neurosci. 2016;44:2072-83.

26. Uvnäs-Moberg K. Oxytocin may mediate the benefits of positive social interaction and emotions. Psychoneuroendocrinology. 1998;23:819-35.

27. Walker S, McGlone F. The social brain: neurobiological basis of affiliative behaviours and psychological well-being. Neuropeptides. 2013;47:379-93.

28. Walker S, McGlone F. C-Tactile Afferents: the mediators of oxytocin release during affiliative interaction? Neuropeptides. 2017a;65:135.

29. Klein H, Elifson KW, Sterk CE. Young adult ecstasy users' enhancement of the effects of their ecstasy use. J Psychoact drugs. 2009;41:113-20.

30. Morrison I, Löken LS, Minde J, Wessberg J, Perini I, Nennesmo I, et al. Reduced C-afferent fibre density affects perceived pleasantness and empathy for touch. Brain. 2011;134:1116-26.

31. Walker SC, Trotter PD, Woods A, McGlone F. Vicarious ratings of social touch reflect the anatomical distribution \& velocity tuning of C-tactile afferents: a hedonic homunculus? Behav brain Res. 2017b;320:91-96.

32. Bilderbeck AC, McCabe C, Wakeley J, McGlone F, Harris T, Cowen PJ, et al. Serotonergic activity influences the cognitive appraisal of close intimate relationships in healthy adults. Biol psychiatry. 2011;69:720-5.

33. Murphy S, Downham C, Cowen P, Harmer C. Direct effects of diazepam on emotional processing in healthy volunteers. Psychopharmacology. 2008; 199:503.

34. Frewen PA, Dozois DJ, Joanisse MF, Neufeld RW. Selective attention to threat versus reward: meta-analysis and neural-network modeling of the dot-probe task. Clin Psychol Rev. 2008;28:308-38.

35. Domes G, Sibold M, Schulze L, Lischke A, Herpertz SC, Heinrichs M. Intranasal oxytocin increases covert attention to positive social cues. Psychol Med. 2013;43:1747-53.

36. Kim Y-R, Oh S-M, Corfield F, Jeong D-W, Jang E-Y, Treasure J. Intranasal oxytocin lessens the attentional bias to adult negative faces: a double blind within-subject experiment. Psychiatry Investig. 2014;11:160-6.

37. Thompson M, Callaghan P, Hunt G, Cornish J, McGregor I. A role for oxytocin and $5-\mathrm{HT}-1 \mathrm{~A}$ receptors in the prosocial effects of 3,4 methylenedioxymethamphetamine ("ecstasy"). Neuroscience. 2007;146:509-14.

38. American Psychiatric Association. Diagnostic and statistical manual of mental disorders (DSM-5 $\left.{ }^{\circledR}\right)$. American Psychiatric Pub, 2013.

39. Mayo LM, Fraser D, Childs E, Momenan R, Hommer DW, de Wit $H$, et al. Conditioned preference to a methamphetamine-associated contextual cue in humans. Neuropsychopharmacology. 2013;38:921-9.

40. Bershad AK, Weafer JJ, Kirkpatrick MG, Wardle MC, Miller MA, de Wit H. Oxytocin receptor gene variation predicts subjective responses to MDMA. Soc Neurosci. 2016b;11:592-9.

41. Wardle MC, Kirkpatrick MG, de Wit H. 'Ecstasy'as a social drug: MDMA preferentially affects responses to emotional stimuli with social content. Soc Cogn Affect Neurosci. 2014b;9:1076-181.

42. Kirkpatrick MG, Lee R, Wardle MC, Jacob S, de Wit H. Effects of MDMA and intranasal oxytocin on social and emotional processing. Neuroopsychopharmacology. 2014;39:1654-63.

43. Ackerley R, Wasling HB, Liljencrantz J, Olausson H, Johnson RD, Wessberg J. Human C-tactile afferents are tuned to the temperature of a skin-stroking caress. J Neurosci. 2014;34:2879-83.

44. Triscoli C, Olausson H, Sailer U, Ignell H, Croy I. CT-optimized skin stroking delivered by hand or robot is comparable. Front Behav Neurosci. 2013;7:208.

45. Dimberg U, Thunberg M, Elmehed K. Unconscious facial reactions to emotional facial expressions. Psychol Sci. 2000;11:86-89.

46. Lang PJ, Greenwald MK, Bradley MM, Hamm AO. Looking at pictures: affective, facial, visceral, and behavioral reactions. Psychophysiology. 1993;30:261-73.

47. Garner M, Mogg K, Bradley BP. Orienting and maintenance of gaze to facial expressions in social anxiety. J Abnorm Psychol. 2006;115:760.

48. Goeleven E, De Raedt R, Leyman L, Verschuere B. The Karolinska directed emotional faces: a validation study. Cogn Emot. 2008;22:1094-118.

49. Lim J, Dinges DF. A meta-analysis of the impact of short-term sleep deprivation on cognitive variables. Psychol Bull. 2010;136:375.

50. Croy I, Luong A, Triscoli C, Hofmann E, Olausson H, Sailer U. Interpersonal stroking touch is targeted to $C$ tactile afferent activation. Behav Brain Res. 2016;297:37-40.

51. Morrison I. ALE meta-analysis reveals dissociable networks for affective and discriminative aspects of touch. Hum brain Mapp. 2016;37:1308-20. 
52. Bjornsdotter M, Lebedev A, Roseman L, Erritzoe D, Feilding A, Petrovic $P$, et al. Effects of acute 3,4-methyle nedioxymethamphetamine (MDMA) on tactile network connectivity. Hum Brain Mapp. 2017; Vancouver, BC.

53. Winocur E, Gavish A, Voikovitch M, Emodi-Perlman A, Eli I. Drugs and bruxism: a critical review. J Orofac pain. 2003;17:99-111.

54. Morley KC, Arnold JC, McGregor IS. Serotonin (1A) receptor involvement in acute 3, 4-methylenedioxymethamphetamine (MDMA) facilitation of social interaction in the rat. Prog NeuroPsychopharmacol Biol Psychiatry. 2005;29:648-57.

55. Preller KH, Pokorny T, Hock A, Kraehenmann R, Stämpfli P, Seifritz E, et al. Effects of serotonin $2 \mathrm{~A} / 1 \mathrm{~A}$ receptor stimulation on social exclusion processing. Proc Nat Acad Sci USA. 2016;113(18):5119-24.

56. Bilderbeck AC, Wakeley J, Godlewska BR, McGlone F, Harris T, Cowen PJ, et al Preliminary evidence that sub-chronic citalopram triggers the reevaluation of value in intimate partnerships. Soc Cogn Affect Neurosci. 2013;9:1419-25.

57. Dumont G, Sweep F, Van der Steen R, Hermsen R, Donders A, Touw D, et al. Increased oxytocin concentrations and prosocial feelings in humans after ecstasy (3, 4-methylenedioxymethamphetamine) administration. Soc Neurosci. 2009:4:359-66.

58. Hysek CM, Domes G, Liechti ME. MDMA enhances "mind reading" of positive emotions and impairs "mind reading" of negative emotions. Psychopharmacology. 2012a;222:293-302.

59. Kirkpatrick MG, Francis SM, Lee $R$, de Wit $H$, Jacob S. Plasma oxytocin concentrations following MDMA or intranasal oxytocin in humans. Psychoneuroendocrinology. 2014a;46:23-31.

60. Wolff K, Tsapakis E, Winstock A, Hartley D, Holt D, Forsling M, et al. Vasopressin and oxytocin secretion in response to the consumption of ecstasy in a clubbing population. J Psychopharmacol. 2006;20:400-10.

61. Kuypers KP, de la Torre R, Farre M, Yubero-Lahoz S, Dziobek I, Van den Bos W, et al. No evidence that MDMA-induced enhancement of emotional empathy is related to peripheral oxytocin levels or $5-\mathrm{HT}$ 1a receptor activation. PLoS ONE. 2014;9:e100719.

62. Ellingsen D-M, Wessberg J, Chelnokova O, Olausson H, Laeng B, Leknes S. In touch with your emotions: oxytocin and touch change social impressions while others' facial expressions can alter touch. Psychoneuroendocrinology. 2014;39:11-20.

63. Scheele D, Kendrick KM, Khouri C, Kretzer E, Schläpfer TE, Stoffel-Wagner B, et al. An oxytocin-induced facilitation of neural and emotional responses to social touch correlates inversely with autism traits. Neuropsychopharmacology. 2014;39:2078.

64. Leng G, Ludwig M. Intranasal oxytocin: myths and delusions. Biol psychiatry. 2016;79:243-50.

65. Bershad AK, Kirkpatrick MG, Seiden JA, de Wit H. Effects of acute doses of prosocial drugs methamphetamine and alcohol on plasma oxytocin levels. J Clin Psychopharmacol. 2015;35:308-12.

66. Liechti ME, Vollenweider FX. Which neuroreceptors mediate the subjective effects of MDMA in humans? A summary of mechanistic studies. Hum Psychopharmacol: Clin Exp. 2001;16:589-98.

67. Liechti ME, Baumann C, Gamma A, Vollenweider FX. Acute psychological effects of 3, 4-methylenedioxymethamphetamine (MDMA, “Ecstasy") are attenuated by the serotonin uptake inhibitor citalopram. Neuropsychopharmacology. 2000;22:513.

68. Farré M, Abanades S, Roset PN, Peiro AM, Torrens M, O'Mathúna B, et al. Pharmacological interaction between 3, 4-methylenedioxymethamphetamine (ecstasy) and paroxetine: pharmacological effects and pharmacokinetics. J Pharmacol Exp Ther. 2007;323:954-62.

69. Hysek C, Simmler L, Ineichen M, Grouzmann E, Hoener M, Brenneisen R, et al. The norepinephrine transporter inhibitor reboxetine reduces stimulant effects of MDMA ("ecstasy") in humans. Clin Pharmacol Ther. 2011;90:246-55.
70. Hysek CM, Simmler LD, Nicola VG, Vischer N, Donzelli M, Krähenbühl S, et al. Duloxetine inhibits effects of MDMA ("Ecstasy") in vitro and in humans in a randomized placebo-controlled laboratory study. PloS ONE. 2012b;7 e36476.

71. Mithoefer MC, Wagner MT, Mithoefer AT, Jerome L, Doblin R. The safety and efficacy of \pm 3 , 4-methylenedioxymethamphetamine-assisted psychotherapy in subjects with chronic, treatment-resistant posttraumatic stress disorder: the first randomized controlled pilot study. J Psychopharmacol. 2011;25:439-52.

72. Danforth AL, Grob CS, Struble C, Feduccia AA, Walker N, Jerome L, et al Reduction in social anxiety after MDMA-assisted psychotherapy with autistic adults: a randomized, double-blind, placebo-controlled pilot study. Psychopharmacology. 2018;235:3137-48.

73. Danforth AL, Struble CM, Yazar-Klosinski B, Grob CS. MDMA-assisted therapy: a new treatment model for social anxiety in autistic adults. Prog NeuroPsychopharmacol Biol Psychiatry. 2016;64:237-49.

74. Cascio C, McGlone F, Folger S, Tannan V, Baranek G, Pelphrey KA, et al. Tactile perception in adults with autism: a multidimensional psychophysical study. J autism Dev Disord. 2008;38:127-37.

75. Kaiser MD, Yang DY-J, Voos AC, Bennett RH, Gordon I, Pretzsch C, et al. Brain mechanisms for processing affective (and nonaffective) touch are atypical in autism. Cereb Cortex. 2015;26:2705-14.

76. Voos AC, Pelphrey KA, Kaiser MD. Autistic traits are associated with diminished neural response to affective touch. Soc Cogn Affect Neurosci. 2012;8:378-86.

77. Dziobek I, Rogers K, Fleck S, Bahnemann M, Heekeren HR, Wolf OT, et al. Dis sociation of cognitive and emotional empathy in adults with Asperger syndrome using the Multifaceted Empathy Test (MET). J autism Dev Disord. 2008;38:464-73.

78. Hysek C, Schmid Y, Simmler L, Domes G, Heinrichs M, Eisenegger C, et al. MDMA enhances emotional empathy and prosocial behavior. Soc Cogn Affect Neurosci. 2014;9:1645-52.

79. Kuypers KP, Dolder PC, Ramaekers JG, Liechti ME. Multifaceted empathy of healthy volunteers after single doses of MDMA: a pooled sample of placebocontrolled studies. J Psychopharmacol. 2017;31:589-98.

80. Wardle MC, de Wit H. MDMA alters emotional processing and facilitates positive social interaction. Psychopharmacology. 2014a;231:1-11.

81. Bishop SJ. Neurocognitive mechanisms of anxiety: an integrative account. Trends Cogn Sci. 2007;11:307-16.

82. Rude SS, Wenzlaff RM, Gibbs B, Vane J, Whitney T. Negative processing biases predict subsequent depressive symptoms. Cogn Emot. 2002;16:423-40.

83. Litz BT, Orsillo SM, Kaloupek D, Weathers F. Emotional processing in posttraumatic stress disorder. J Abnorm Psychol. 2000;109:26.

84. Mithoefer M, Wagner M, Mithoefer A, Jerome L, Martin S, Yazar-Klosinski B. Durability of improvement in PTSD symptoms and absence of harmful effects or drug dependency after MDMA-assisted psychotherapy: a prospective long-term follow-up study. J Psychopharmacol. 2013;27:28-39.

85. Oehen $\mathrm{P}$, Traber R, Widmer V, Schnyder U. A randomized, controlled pilot study of MDMA ( \pm 3 , 4-Methylenedioxymethamphetamine)-assisted psychotherapy for treatment of resistant, chronic Post-Traumatic Stress Disorder (PTSD). J Psychopharmacol. 2013;27:40-52.

86. Wagner MT, Mithoefer MC, Mithoefer AT, MacAulay RK, Jerome L, YazarKlosinski $B$, et al. Therapeutic effect of increased openness: Investigating mechanism of action in MDMA-assisted psychotherapy. J Psychopharmacol. 2017:31:967-74.

87. Kirk J, Doty P, De Wit H. Effects of expectancies on subjective responses to oral $\Delta$ 9-tetrahydrocannabinol. Pharmacol Biochem Behav. 1998;59:287-93.

88. Mitchell SH, Laurent $\mathrm{CL}$, De Wit $\mathrm{H}$. Interaction of expectancy and the pharmacological effects ofd-amphetamine: subjective effects and self-administration. Psychopharmacology. 1996;125:371-8. 